\title{
Konkurrierende Übersetzungen im Vergleich: Heimito von Doderers Kurzgeschichten „Léon Pujot“ und „Begegnung im Morgengrauen“ auf Französisch
}

\begin{abstract}
Heimito von Doderers Kurzgeschichten „Léon Pujot“ und „Begegnung im Morgengrauen“ wurden je zweimal ins Französische übertragen. Der Beitrag vergleicht die vorliegenden Übersetzungen dieser Texte miteinander, beschreibt die angewandten Übersetzungsverfahren und -strategien und analysiert die je daraus resultierenden Übersetzungen an konkreten Beispielen.
\end{abstract}

Keywords: Heimito von Doderer, Übersetzung, Französisch, Übersetzungsvergleich, Kurzgeschichte, Skopostheorie

Heimito von Doderers Kurz- und Kürzestgeschichten, zu denen die hier näher betrachteten Geschichten „Léon Pujot“ und „Begegnung im Morgengrauen“ zählen, standen im französischen Sprachraum lange, wie der Übersetzer Raymond Voyat $\mathrm{zu}$ bedenken gibt, der sich ihrer schlussendlich angenommen hat, im Schatten seiner „romans viennois“ (Voyat 1995, 61), der großen Wien-Romane. Dabei verteidigen Doderers Kurzgeschichten durchaus ihren Platz in seinem Werk, auch wenn ihnen häufig eine handlungsorientierte Banalität vorgeworfen wurde - eine Kritik, der Doderer selbst entgegentritt: „Wer ein wirklicher und wirksamer Geschichtenerzähler ist, der erweckt immer den Eindruck, als sei nichts dabei. Aber man versuche es einmal!“ (Doderer 2001b, 50)

Die „epische Breite in der Westentasche“ (Doderer 2001a, 15), wie Doderer die paradoxe Eigenheit der Kurzgeschichte in einem Brief an Magdalena Brückner vom 4. Mai 1949 beschreibt, und mit ihr die Ausflüge ins Kriminalistische und Abenteuerliche, gehören, wie der Autor bestätigt, „eben auch zu meiner Palette“ (Doderer 2001a, 20). Gerade das Beharren auf „kräftige[n] Plots“ ist das verbindende Element der zwischen verschiedensten „Bauformen und Stillagen“ (LoewCadonna 1990, 231) wechselnden Dodererschen Kurzprosa. Besonders in den „überraschungsreichen stories“ (Loew-Cadonna 1990, 234), wie etwa „Léon Pujot“ und „Begegnung im Morgengrauen“, mit ihrem ausgiebigen Gebrauch von plötzlichen Ereignissen und „fatale[n] Requisit[en]“ (Schmidt-Dengler 1995a, 503) lässt sich dieser spezifische Fokus auf den Plot beobachten. Die Kurz- und Kürzestgeschichten als ,ironischer Kontrapost zur Großform des Romans“ (Schmidt-Den- 
gler 1995a, 503) sind nicht anders als die Romane gleichfalls der Doderer zugeschriebenen ,Fatologie', und damit zusammenhängend dem Metaphernfeld des Zuges, der Geleise und der Weichen sowie des „Zusammenpralls“ (Loew-Cadonna 1990, 241), geschuldet:

Die Umwege und Bögen der Dodererschen Fabeln bestehen gleichsam aus je voneinander abgewinkelten Kurz- oder Langstrecken. Dazu paßt es, wenn der Erzähler [...] die Metaphern von Geleise und Weiche bemüht: zuerst surrt der Zug des Lebens trutzig und putzig auf seiner Bahn dahin, dann klappt jählings die Weiche, und es beginnt ein neuer Bahnabschnitt. (Loew-Cadonna 1990, 244)

Über die Gattung Kurzgeschichte und das Motiv Zug wird auch die Verbindung zu einem weiteren zentralen Punkt Dodererscher Textgestaltung hergestellt: jenem der Apperzeption, des bewussten Wahrnehmens als tableauartige Momentaufnahme im Sinne der von Doderer oft gebrauchten Formel von der ,Anatomie des Augenblicks'. So entstehen Beschreibungen von Wahrnehmungen, die zwar statisch scheinen, die „aber aus dem gesamten Zusammenhang eine unheimliche Dynamik“" (Schmidt-Dengler 2001, 206) entfalten.

Während der Zug und die rasante Fahrt als Motive die Wahrnehmungsbedingungen im wörtlichen Sinn verändern und im übertragenen neue Perspektiven jenseits der eingefahrenen Geleise des Alltags eröffnen, bringt Doderer auch die Entstehung der Kurzgeschichte mit einer Veränderung der Wahrnehmung in Verbindung:

Der Erzähler setzt den wesentlichen Akt, noch bevor wir ihm zusehen können, also bevor er uns eine Geschichte erzählt. Plötzlich fällt der stets umgebende Ringwulst seines Alltags an einer Stelle ein, es entsteht eine bisher nicht gekannte Seh-Scharte, durch sie pfeift schon der Wind des Einfalles, und die Geschichte tritt herein [...]. (Doderer 2001b, 50)

In dieser Hinsicht sind die Geschichten „Léon Pujot“ und „Begegnung im Morgengrauen“ geradezu Musterbeispiele des Dodererschen Verständnisses von Kurzgeschichten und - in Verbindung mit der „Eisenbahn“ als „eines der zentralen metaphorischen Substrate“ (Schmidt-Dengler 2004, 377) - für den Moment der Apperzeption. ${ }^{1}$

1 Züge und Zugfahrten spielen etwa eine zentrale Rolle in Die Strudlhofstiege (1951), Die Wasserfälle von Slunj (1963) oder Ein Mord, den jeder begeht (1938). Dabei dient das Bild des (fahrenden) Zuges häufig auch der Charakterisierung von Figuren, etwa um die ,Fahrplanmäßigkeit‘ eines Charakters zu illustrieren, oder auch als die Handlung antreibendes Element. In den Wasserfällen von Slunj fungiert eine Spielzeugeisenbahn als eine Art ,Erinnerungszug in die Vergangenheit‘ (vgl. Schneider 1985, 79). 
„Léon Pujot“, 1929 unter dem Eindruck eines Zeitungsberichts entstanden und 1930 in der Wiener Zeitung Der Abend veröffentlicht (vgl. Schmidt-Dengler 1995b, 508f.), ist die abenteuerliche Geschichte des Taxifahrers Léon Pujot. Ihm gelingt es in einem waghalsigen Manöver, von seinem Taxi aus auf die Lokomotive eines führerlos dahinfahrenden Schnellzuges aufzuspringen und diesen zu stoppen. Entscheidend für den Erfolg von Pujots Handlung ist es, dass er den richtigen Zeitpunkt erkennt: Denn der Moment seiner Rettungstat ist zugleich der, in dem es für den sozusagen neben dem Zug des Lebens fahrenden Pujot an der Zeit ist, auf das richtige Gleis zu wechseln und sein Leben (ebenso wie er den Zug unter seine Kontrolle bringt) selbst in die Hand zu nehmen, und mithin der Augenblick seiner „zweite[n] Geburt“ (Doderer 1995b, 266), die nur wenige von Doderers Figuren erreichen.

Auch in „Begegnung im Morgengrauen“, 1933 entstanden (vgl. Schmidt-Dengler 1995b, 508), steht eine Zugfahrt im Zentrum der Geschichte: Im Winter 1916 fährt ein junger Offizier, noch betrunken von einem Abschiedsgelage, mit einem Zug zurück in Richtung seines Einsatzortes an der Front. In seinem Abteil trifft er, benebelt vom Rausch, auf einen Henker, den er zunächst für einen harmlosen Reisenden, einen „,gemütliche[n] Sachse[n]““ (Doderer 1995a, 255), hält, der mit seinem Richtbeil zu einer Exekution nach Köln reist. Infolge dieser Begegnung gelangt der Leutnant zu einem Moment der Klarheit, die Zugfahrt ist eine unaufhaltsame Annäherung an den Tod, an jenen, den der Henker um 7:30 am Morgen herbeizuführen hat, und zugleich an den an der Front jederzeit drohenden Soldatentod. Rund zwei Jahrzehnte später hat Doderer dementsprechend das Reisen als Todesmetapher verstanden: „Reisen, migrare, ist ein Vorbild des Todes. Auch bedeuten Reiseträume unser Denken an den Tod. Abreisen heißt, alles liegen und stehn lassen, schnittweis, integral.“ (Doderer 1969, 22)

Von beiden Kurzgeschichten gibt es je zwei Übersetzungen ins Französische. Die zuerst entstandenen finden sich jeweils in einem Band mit ,Contes Allemands', einmal in der ,Deutschen Reihe für Ausländer‘ des Hueber Verlags, das andere Mal im Rahmen der Reihe ,dtv zweisprachig‘, die beide - gedacht für Deutschlernende - den deutschen Text und seine französische Übersetzung einander gegenüberstellen: „Begegnung im Morgengrauen“ im Hueber-Band aus dem Jahr 1964, übersetzt von Bernadette Sindezingue und Jean Prévot, und „Léon Pujot“ im Pendant des Deutschen Taschenbuch Verlags aus dem Jahr 1978, übertragen von Gérard Peterolff. Diese primär didaktischen Zwecken dienenden und der Form des Paralleltexts geschuldeten Übersetzungen wurden erst 1998 um jene des bereits erwähnten Übersetzers und Germanisten Raymond Voyat ergänzt, die im Rahmen seiner Übersetzung der Kurz- und Kürzestgeschichten im Pariser Verlag Périple erschienen (2008 neu aufgelegt vom Verlag Motifs, Monaco). 
Während die Übersetzungen von 1964 und 1978 sehr genau Doderers Ausgangstext folgen und neben einer möglichst großen semantischen auch eine lautliche und syntaktische Annäherung an das deutsche Original anstreben, verfolgt Voyat, unter Berufung auf Doderer selbst, einen freieren Ansatz, erklärt dieser doch im Repertorium, dass eine Übersetzung „nicht nur dem Original mit Liebe und Sorgfalt nachkommen und nahekommen“ müsse, sondern dem Ausgangstext auch „,voraus sein“ solle: „Sie muß einen ganz neuen Reiz des Werkes sichtbar machen, der latent in ihm war, aber in der ursprünglichen Sprache nicht so sehr zur Geltung gelangen konnte. Jede wirkliche und wirksame Übertragung wird in irgendeiner Hinsicht das Original übertreffen.“ (Doderer 1969, 251)

Voyat, der unter anderem auch Novalis’ Hymnen an die Nacht und Goethes Römische Elegien ins Französische übertragen hat und bereits in den 1980er Jahren mit der Übersetzung der Kurz- und Kürzestgeschichten begann, sieht seine Verantwortung als Übersetzer vor allem dem Lesepublikum gegenüber, und dies im Sinne der Skopostheorie von Reiß/Vermeer, welche die Notwendigkeit der Äquivalenz von Ausgangstext und Übersetzung infrage stellt und das kommunikative Ziel der Übersetzung in den Vordergrund rückt (vgl. Pym 2010, 44). Für Voyat, der sich ausdrücklich auf die Skopostheorie beruft (vgl. Voyat 2004, 389), ist es zwar wichtig, die Eigenheiten eines Textes zu vermitteln, gleichzeitig sei aber zu bedenken, „daß dem zu übersetzenden Schriftsteller im Hinblick auf seine Rezeption in einer anderen Sprache auch geholfen werden muß, kulturelle und sprachliche Barrieren zu überwinden“, wozu es abträglich sei, „wenn alle Eigenwilligkeiten und Schreibticks unbarmherzig nachvollzogen“ (Voyat 2004, 389) würden. Im Fall einer Doderer-Übersetzung ins Französische werde die Vermittlung zusätzlich durch die französische Literaturtradition sowie die daraus resultierenden Lesegewohnheiten erschwert, die - so Voyat weiter - vor allem bei Prosa „großen Wert auf Kürze, Einfachheit, Einmaligkeit und Unmißverständlichkeit“ (Voyat 2004, 389) lege.

Abgesehen davon, dass es vermutlich mindestens so viele Belege für wie gegen diese Behauptung gibt, und abgesehen davon, dass der Vorwurf der Kompliziertheit zwar möglicherweise auf Doderers Romane, weniger jedoch auf seine Kurz- und Kürzestgeschichten zutrifft, unterstellt Voyat dem französischen Lesepublikum hier, von Doderers Prosa überfordert zu sein, die, wie er meint, „im Regelfall eher weitschweifig und verschachtelt sowie wortreich und nicht selten doppeldeutig, oder mit einem Wort: barock“ (Voyat 2004, 389) sei. Voyat verfolgt eine Übersetzungsstrategie im Sinne der Lesbar- und Zugänglichkeit und grenzt sich so dezidiert von „Übersetzerkollegen [ab], die Doderer mit höchster Akribie ins Französische übertragen haben“ (Voyat 2004, 390), und damit zugleich von denen, die sich vor ihm an „Léon Pujot“ und „Begegnung im Morgengrauen“ versucht haben. Denn zu „den Aufgaben der Übersetzer“ zähle vor allem auch, „ih- 
ren Autoren neue Leserkreise zu erschließen“, weshalb eine Übertragung „nicht abschreckend wirken“ (Voyat 2004, 390) solle, sondern den Zugang zum Text generell erleichtern müsse - insbesondere im Falle Doderers:

Doderer ist nun einmal [...] ein Magier des Wortes, ein belesener, von der Etymologie und der Geschichtsschreibung faszinierter Intellektueller, der im Original schon nicht immer gemeinhin verständlich ist. Es gehört also auch zur Aufgabe des Übersetzers, dem Leser den Einstieg in eine solche Welt zu erleichtern - und nicht zu erschweren. (Voyat 2004, 389)

Es soll hier jedoch nicht der Eindruck entstehen, als hätte man nach Voyats Verständnis freie Hand, beim Übersetzen mit dem Ausgangstext zu verfahren, wie es einem beliebt. Auch Voyat ist bemüht, Doderer gerecht zu werden - allerdings nicht um jeden Preis: Für ihn liegt die Schwierigkeit in der „Gestaltung eines vertretbaren Ausgleichs“ (Voyat 2004, 389) zwischen Textentsprechung und Zugeständnissen an das Lesepublikum. Ein Spannungsverhältnis, das sich auch in den Übersetzungen von „Léon Pujot“ und „Begegnung im Morgengrauen“ zeigt und das gerade im Vergleich mit den früheren Übersetzungen, die näher am Ausgangstext bleiben, deutlich wird.

Eingriffe, die (anscheinend) im Sinne einer Erleichterung der Rezeption vorgenommen wurden, zeigen sich insbesondere bei Voyats Umgang mit Unbestimmtheiten und (vermeintlichen) Unklarheiten, die er in „Begegnung im Morgengrauen“ zugunsten einer besseren Lesbarkeit häufig tilgt. So lautet der Beginn des ersten Satzes, eine Zeitangabe, die in ihrer Vagheit den benebelten Zustand des Offiziers nach dem Abschiedsgelage widerspiegelt: „Es mochte zwischen zwei und drei Uhr morgens sein [...]“ (Doderer 1995a, 255). Von Sindezingue und Prévot - wie gesagt, sehr nahe am Ausgangstext - wird dies mit „Il pouvait être entre deux heures et trois heures du matin [...]“ (Doderer 1964, 69) übersetzt, wohingegen Voyat sich für ein durchaus konkretes „Vers deux heures et demie du matin [...]“ (Doderer 2008a, 74) entscheidet und so den unwirklichen, an eine Geistergeschichte erinnernden Moment, den Doderer mit der Zeitangabe gleich zu Beginn erzeugt, eliminiert.

Rezeptionserleichterungen erreicht Voyat auch, indem er gelegentlich kurze Erläuterungen einschiebt bzw. hervorhebt oder komplexe Ausdrucksweisen vereinfacht. Dabei scheint ihn weniger zu interessieren, dass Letztere, die in Doderers Kurz- und Kürzestgeschichten ohnehin selten anzutreffen sind, natürlich auch eine Funktion erfüllen - etwa wenn der Offizier bei dem Gedanken an die Exekution eines Unbekannten durch seinen Reisegenossen in eine eigentümliche Mischung aus Umgangssprache und bürokratische Umständlichkeit verfällt: „,So'n Brimborium von wegen einem einzigen Kerl, der da umgebrungen werden sollte, erschien mir dazumalen, bei den sonst obwaltenden Umständen, als nicht recht angebracht.““ (Doderer 1995a, 257) Sindezingue und Prévot, die sich hier so- 
wohl um syntaktische als auch um lautliche Entsprechung bemühen, übersetzen diesen Gedanken des Offiziers wie folgt: ,,,Un tel brimborion à cause d'un seul coquin, qui devait alors être occis, me semblait à ce moment-là, dans cet état de choses, pas tout à fait à sa place...““ (Doderer 1964a, 75) Im Gegensatz dazu überträgt Voyat viel geradliniger und vereinfacht die Syntax, wodurch nicht nur die komplizierte Struktur des Satzes aufgehoben, sondern eben auch die Umständlichkeit als stilistisches Merkmal getilgt wird: „,Et vu les circonstances de l'époque, je trouvais assez indécent tout ce tralala pour un seul mec qu'il fallait raccourcir.“" (Doderer 2008a, 77)

Auch die Beschreibung der Landschaft, die durch die von der Fahrt des Zuges, aber auch von einem nur langsam abklingenden Rausch und der Erwartung der Front beeinflusste Wahrnehmung des Offiziers einen vage irrealen und bedrohlichen Charakter annimmt, wird von Voyat im Sinne einer Vereindeutigung verkürzt. Bei Doderer lautet die betreffende Passage komplett:

Der Tag war herauf; das Grau des Morgens verkroch sich in den Boden, sank nach rückwärts, talab, ins tiefer gelegene Land, woher man gefahren kam. Es legte sich auch in irgendwelche Winkel der Seele nieder, zusammen mit den Resten eines verdunstenden Rausches, die da oder dort noch hängen geblieben waren... darüber stand ein leerer Raum, zwischen Zwerchfell und Herz etwa, er stand sozusagen klaffend bereit, eine ungewisse Zukunft aufzunehmen. Im Rücken spürte man gerade das mächtige Wirken der Schubmaschine [...]. (Doderer 1995a, 258)

Bei Voyat ist die Landschaftsbeschreibung dagegen um einiges simpler geraten, so fehlt etwa die dreifache Betonung des Zurückliegenden - „rückwärts, talab, ins tiefer gelegene Land“ -, und darüber hinaus wird die „Seele“ zur einfacher zu greifenden ,Erinnerung“ (,souvenir“): „L'aube pointait, la grisaille du petit matin collait à la terre tout au fond de la vallée qu'on laissait derrière soi. Ainsi en est-il des recoins du souvenir, alors que vont se dissipant les restes d'une ivresse...“ (Doderer 2008a, 79)

Im Sinne der einfacheren Zugänglichkeit nimmt Voyat auch Änderungen im Aufbau einzelner Abschnitte vor, was sich auf die von Doderer in dieser Kurzgeschichte gerne eingesetzten Retardierungen auswirkt - etwa bei der Enthüllung des „fatale[n] Requisit[s]“ (Schmidt-Dengler 1995a, 503) der Geschichte, dem Richtbeil. Bei Doderer folgt auf die Frage des betrunkenen Offiziers an den mitreisenden Henker, den er zu diesem Zeitpunkt aufgrund des mitgeführten Etuis noch für einen Musiker hält, eine recht ausführliche Beschreibung des Mannes:

„Hören Sie‘, fing der Leutnant wieder an, ,was ist denn aber nun das für’n Ding?‘ Das Sprechen machte ihm etwas Mühe, die Zunge hatte sozusagen eine leichte Schlagseite. Der Herr gegenüber trug einen jener Stehkragen, die sich wie ein niedriges, gleichmäßiges Ränftchen 
um den Hals legten, ohne Ecken oder Spitzen, fast wie ein Priester. Dazu Maschinschlips. ,Sächsischer Musiker', dachte Herr von E. verschwommen.

,Das is'n Richtbeil, da oben‘, sagte jetzt der ,Sachse‘. (Doderer 1995a, 256)

Voyat dagegen verschiebt die Beschreibung des Scharfrichters nach vorne, übrig bleibt nur der Dialog: „Dites voir, reprit le lieutenant, c'est quoi votre truc? Il avait la langue pâteuse et les mots lui venaient avec difficulté. - Là-haut? C'est une hache de bourreau." (Doderer 2008a, 76) Durch Voranstellen der Schilderung des Henkers wird der Spannungsbogen der Passage verändert und auch die Assoziation von „Priester“ und „,Richtbeil““ aufgeweicht. Gerade solche Konstruktionen aber sind es, auf denen Doderers Methoden der „literarische[n] Effekterzeugung“ (Loew-Cadonna 1990, 232) basieren. Nicht nur das, was gesagt wird, entfaltet seine Wirkung in der Erzählung, sondern eben auch das, was nicht gesagt wird: „Elemente also, die auf sinnfälligen Lücken der Leserinformation, auf verzögerter Turbulenz, auf der List der Andeutung gründen.“ (Loew-Cadonna 1990, 232) Wenn Voyat diese Lücken und Andeutungen nun verkürzt oder tilgt, wirkt er auch den von Doderer sehr bewusst eingesetzten und konstruierten Effekten der Spannungssteigerung entgegen (vgl. Loew-Cadonna 1990, 233).

Ähnliches lässt sich auch am Anfang von „Léon Pujot“ feststellen: Der Taxifahrer Pujot befindet sich, nachdem er einen Stammgast nach Paris chauffiert hat, auf einer Leerfahrt zurück nach Nancy - geschildert wird das Hintersichlassen der Stadt und das Hineinfahren in die offene Landschaft, ein Kontrast von „Auseinanderklaffen und Zueinanderfließen“ (Voyat 2004, 387), wie es auch Voyat als typisch für Texte Doderers beschreibt. Zu Beginn der Kurzgeschichte zeige sich, so Voyat, ,wie sich die geschlossene Stadtwelt allmählich lockert und in Landschaft auflöst“ (Voyat 2004, 387), was sich in den ersten Sätzen von „Léon Pujot“ ganz ähnlich liest: ,[S]ie fuhren mit stürmendem Motor gegen die östlichen Vorstädte hinaus und über die Stadtgrenze, bis Häuser, Schuppen und Geleise ganz aufgelöst zurückblieben und weiterhin der Flußlauf der Marne sichtbar ward.“ (Doderer 1995b, 259)

Gérard Peterolffs Übersetzung aus dem Jahr 1978 bemüht sich hier um größtmögliche Entsprechung - zumindest der Oberfläche. Die Stelle lautet: „[I]ls partaient, le moteur furieusement lancé, vers les faubourgs est et par delà les limites de la ville, jusqu'à ce que les maisons, les entrepôts et les rails fussent restés en arrière, entièrement dissouts, et que la cours de la Marne devint visible plus loin.“ (Doderer 1978, 137)

Wenn hier dem Ausgangstext entsprechend der Übergang von der Stadt zum Land und das Auflösen der Landschaft in der Bewegung vermittelt wird, bleibt bei Voyat - entgegen seiner eigenen Analyse - nur noch der eher sachlich geratene Stadt-Land-Kontrast übrig, unter anderem auch durch das Ersetzen von Doderers 
mitunter blumigen, aber ausdrucksstarken Adjektiven durch neutralere Wendungen. In dieser Passage etwa wird der „stürmende Motor“ der Vorlage zum nüchternen ,Vollgas“ („,à plein gaz“): „Et les voici partis à pleins gaz en direction de la banlieue est, laissant bientôt derrière eux Paris, ses hangars, ses rails, et ses ultimes maisons, à mesure qu'ils approchaient de la Marne.“ (Doderer 2008b, 81f.)

Auch die Dynamik der Wahrnehmung, die bei Doderer durch die Geschwindigkeit der Fortbewegung in Zug oder Auto entsteht, lässt Voyat unter den Tisch fallen. Doderer beschreibt neuerlich einen Übergang, in diesem Fall den von einer ländlichen zu einer städtischen Umgebung entlang der gerade befahrenen Bahntrasse, und zwar in eben dem schnellen Wechsel, wie er von Léon Pujot wahrgenommen wird, der nun als Lokomotivführer agiert: „Jetzt aber, in dem grünen Wogen der Landschaft, erschienen Zacken und gerade Striche, erschienen Dächer, Türme, eine Häuserzeile sprang in's Blickfeld, lief weithin.“ (Doderer 1995b, 264) Auch in der Übersetzung von Peterolff sind diese in Bewegung überführte Landschaft und die besondere Art der Wahrnehmung vom Führerstand einer schnell dahinfahrenden Lokomotive aus nachvollziehbar: „Mais dans la houle verte du paysage apparaissaient maintenant des dents et des lignes droites, des toits et des tours, une rangée de maison sauta dans le champ visuel et en ressortit.“ (Doderer 1978, 149) Bei Voyat hingegen bleibt die Beschreibung der Landschaft statisch und unbelebt; statt ,ins Blickfeld zu springen' ,zeichnet sie sich ab': „Des traits et des dentelures se dessinèrent dans le vallonnement de la verte campagne, des tours, des toits, puis une rangée de maisons.“ (Doderer 2008b, 88) So nimmt Voyat wie schon in „Begegnung im Morgengrauen“ auch in „Léon Pujot“ ,Vereinfachungen“ vor oder schiebt kurze Ergänzungen ein, um für mehr Klarheit zu sorgen, und verändert die Reihenfolge von Textabschnitten, was in „Léon Pujot“ wohl die Abenteuerhandlung unterstützen und die Spannung steigern soll, teilweise aber wichtige Aspekte von Doderers Prosa, wie jene der dynamischen Wahrnehmung, außen vor lässt.

Auch der Zug, der in „Léon Pujot“ - typisch für Doderers Herangehensweise in den 1930er Jahren - als eine „unbändige[], ja sogar animalische[] Kraft, [...] [als] laute[s], bedrohliche[s] ,Wesen' [...]" (Schneider 1985, 99) beschrieben wird, erscheint in Voyats Übersetzung wie ein gezähmtes Pendant. Er wird zwar bei Voyat gar zum „mastodonte“ (Doderer 2008b, 86), einem Monstrum, das schnaubt und braust, aber einige jener Details, die ihn bei Doderer erst zu einer Art lebendigem Maschinenwesen machen, fallen in Voyats Übersetzung weg. So wird etwa „das runde Maul der Feuerbox“ (Doderer 1995b, 263), von Peterolff noch als „la gueule ronde du foyer“ (Doderer 1978, 147) wiedergegeben, bei Voyat zu einer simplen „la boîte à feu“ (Doderer 2008b, 87). Auch Pujots „Wagen“, dessen vergleichsweise Geringfügigkeit Doderer verdeutlicht, indem er den kümmerlichen Klang seiner Hupe zur Lautstärke der Zugsirene in Beziehung setzt, ist bei 
Voyat einfach nur ein „taxi“: „Es war ein Ton, gegen den sich das Signalhorn seines Wagens wie Ferkelgequieke ausnehmen mochte.“ (Doderer 1995b, 264) Bei Voyat hingegen ist der zwar ein wenig eigentümliche, aber doch aussagekräftige Ferkel-Vergleich weggefallen: „Ça c'était du vrai, comparé au klaxon aigrelet de son taxi!“ (Doderer 2008b, 88)

Abschließend lässt sich feststellen, dass die konkurrierenden Übersetzungen von „Begegnung im Morgengrauen“ und „Léon Pujot“ in beinahe prototypischer Weise als Beispiele für die zwei verschiedenen Modi der Übersetzung nach Juliane House (vgl. House 2015, 65f.) dienen können. Die Übersetzungen für die Paralleltextausgaben von Sindezingue und Prévot bzw. Peterolff erfüllen die Kriterien einer ,offenen' Übersetzung, die durch die enge Bindung an den Ausgangstext als Übersetzung erkennbar bleiben und nicht als eigenständiger Text, als ,zweites Original', rezipiert werden, sondern als Möglichkeit, „den neuen zielsprachlichen Adressaten im Medium einer anderen Sprache Zugang zum Originaltext“ (Zybatow 2008, 32) zu bieten. Das wird in diesem Fall durch die Form der Veröffentlichung, die Übersetzung und Ausgangstext direkt einander gegenüberstellt, noch verstärkt.

Voyat dagegen scheint mit seiner an Zielkultur und vermuteter Rezeptionshaltung orientierten Übersetzungsstrategie eher in Richtung von (nach House) ,verdeckten Übersetzungen“ zu gehen, die „sprachlich so gestaltet [sind], dass sie sich nahtlos in das entsprechende Genre der Zielkultur einpassen und von den Adressaten wie Originale rezipiert werden“ (Zybatow 2008, 32f.). Um dies zu ermöglichen, müssen Veränderungen an sprachlichen Formen und Textmustern vorgenommen werden: Wer einen Text in die Zielsprache überträgt, setzt dafür einen sogenannten ,kulturellen Filter‘ ein, „mit dessen Hilfe er das Original durch die Brille des Zielkulturadressaten sieht und versucht, mit seiner Übersetzung die zielkulturellen konventionalisierten Erwartungen bei [der] Textrezeption zu erfüllen.“ (Zybatow 2008, 33)

So stehen sich hier zwei gegensätzliche Übersetzungsstrategien gegenüber, die beide Stärken und Schwächen haben: Übersetzt die eine im Sinne einer Äquivalenz, die der Oberfläche der Satz- und Textkonstruktion geschuldet ist und die sich zwar sehr nahe am Ausgangstext bewegt, aber die Frage aufwirft, ob die deutsche Satzstruktur für den französischen Text geeignet ist, übersetzt die andere mit Blick auf das Lesepublikum, was mitunter zu Abweichungen und Vereinfachungen führt und gerade das in der Übersetzung zu kurz kommen lässt, was den Reiz von Doderers Prosa ausmacht. 


\section{Literaturverzeichnis}

Doderer, Heimito von. „Begegnung im Morgengrauen / Rencontre à l’áube“. Erzählungen Contes. Dürrenmatt. Andersch. von Doderer. Broch. Hg. Klaus Zobel. Aus dem Deutschen von Bernadette Sindezingue und Jean Prévot. (= Deutsche Reihe für Ausländer, Reihe F: Zweisprachige Reihe, Bd. 3) München: Max Hueber, 1964. 68-79.

Doderer, Heimito von. Repertorium. Ein Begreifbuch von höheren und niederen Lebens-Sachen. Hg. Dietrich Weber. München: Biederstein, 1969.

Doderer, Heimito von. „Léon Pujot“. Deutsche Erzählungen 2 - Contes Allemands 2. Heimito von Doderer. Hugo von Hofmannsthal. Franz Kafka. Robert Musil. Joseph Roth. Rainer Maria Rilke. Arthur Schnitzler. Übersetzt aus dem Deutschen von Gérard Peterolff. München: dtv, 1978. 136-153.

Doderer, Heimito von. „Begegnung im Morgengrauen“. Ders. Die Erzählungen. Hg. Wendelin Schmidt-Dengler. 3., erw. Aufl., München: C. H. Beck, 1995a. 255-259.

Doderer, Heimito von. „Léon Pujot“. Ders. Die Erzählungen. Hg. Wendelin Schmidt-Dengler. 3., erw. Aufl., München: C. H. Beck, 1995b. 259-266.

Doderer, Heimito von. „Drei Briefe Heimito von Doderers zur Kurzprosa. Herausgegeben und kommentiert von Gerald Sommer“. „Schüsse ins Finstere“. Zu Heimito von Doderers Kurzprosa. Hg. Gerald Sommer und Kai Luehrs-Kaiser. Würzburg: Königshausen \& Neumann, 2001a (Schriften der Heimito von Doderer-Gesellschaft; 2). 13-22.

Doderer, Heimito von. „Eisenreichs Einfälle“. „Schüsse ins Finstere“. Zu Heimito von Doderers Kurzprosa. Hg. Gerald Sommer und Kai Luehrs-Kaiser. Würzburg: Königshausen \& Neumann, 2001b (Schriften der Heimito von Doderer-Gesellschaft; 2). 50.

Doderer, Heimito von. „Rencontre au petit matin“. Histoires brèves et ultra-brèves. Nouvelles. Traduit de l'allemand (Autriche) par Raymond Voyat. Monaco: Motifs, 2008a. 74-80.

Doderer, Heimito von. „Léon Pujot“. Histoires brèves et ultra-brèves. Nouvelles. Traduit de l'allemand (Autriche) par Raymond Voyat. Monaco: Motifs, 2008b. 81-91.

House, Juliane. Translation quality assessment: Past and present. London, New York: Routledge, 2015.

Loew-Cadonna, Martin. „Suspense in Doderers Erzählen“. Sprachkunst. Beiträge zur Literaturwissenschaft 21.2 (1990): 231-249.

Pym, Anthony. Exploring Translation Theories. London, New York: Routledge, 2010.

Schmidt-Dengler, Wendelin: „Nachwort des Herausgebers“. Heimito von Doderer: Die Erzählungen. Hg. Wendelin Schmidt-Dengler. 3., erw. Aufl., München: C. H. Beck, 1995a. 499-505.

Schmidt-Dengler, Wendelin. „Anmerkungen“. Heimito von Doderer: Die Erzählungen. Hg. Wendelin Schmidt-Dengler. 3., erw. Aufl. München: C. H. Beck, 1995b. 507-512.

Schmidt-Dengler, Wendelin. „Die Dynamik der stehenden Bilder in Doderers Prosa“. „Schüsse ins Finstere“. Zu Heimito von Doderers Kurzprosa. Hg. Gerald Sommer und Kai LuehrsKaiser. Würzburg: Königshausen \& Neumann, 2001 (Schriften der Heimito von DodererGesellschaft; 2). 203-212.

Schmidt-Dengler, Wendelin. „,Man sollte nie mit dem Automobil über den Semmering fahren“. Die Bahnfahrt als zentrales Motiv bei Heimito von Doderer". Gassen und Landschaften: Heimito von Doderers „Dämonen“ vom Zentrum und vom Rand aus betrachtet. Hg. Gerald Sommer. Würzburg: Königshausen \& Neumann, 2004 (Schriften der Heimito von DodererGesellschaft; 3). 373-381. 
Schneider, Karl Heinrich. Die technisch-moderne Welt im Werk Heimito von Doderers. Frankfurt/ M., Bern, New York: Peter Lang, 1985.

Voyat, Raymond. „Les Austriacismes dans les œuvres mineures de Heimito von Doderer“. Parallèles 17 (1995): 61-67.

Voyat, Raymond. „Der Topos von Stadt und Land in Heimito von Doderers ,Kurz- und Kürzestgeschichten““. Gassen und Landschaften: Heimito von Doderers „Dämonen“ vom Zentrum und vom Rande aus betrachtet. Hg. Gerald Sommer. Würzburg: Königshausen \& Neumann, 2004 (Schriften der Heimito von Doderer-Gesellschaft; 3). 383-391.

Zybatow, Lew. „Literaturübersetzung im Rahmen der Allgemeinen Translationstheorie.“ Im Brennpunkt: Literaturübersetzung. Hg. Wolfgang Pöckl. Frankfurt/M., New York, Wien et al.: Peter Lang, 2008 (Forum Translationswissenschaft; 8). 9-42.

Johanna Lenhart, geb. 1988 in Feldkirch. Studium der Germanistik und Vergleichenden Literaturwissenschaft an der Universität Wien. Seit 2017-19 OeAD-Lektorin an der Al-Alsun Sprachenfakultät der Ain-Shams Universität Kairo (Ägypten). Seit 2020 OeAD-Lektorin an der Masaryk-Universität Brno (Tschechien). 
